C2017, Elsevier. Licensed under the Creative Commons Attribution-NonCommercialNoDerivatives 4.0 International http://creativecommons.org/about/downloads

(1) $\Theta \Theta$

BY No ND 


\title{
Multiscale Segmentation of Exudates in Retinal Images using Contextual Cues and Ensemble Classification
}

\author{
M. Moazam Fraz ${ }^{a^{*}}$, Waqas Jahangir ${ }^{a}$, Saqib Zahid ${ }^{a}$, Mian M. Hamayun ${ }^{a}$, Sarah A. Barman ${ }^{b}$ \\ ${ }^{a}$ School of Electrical Engineering and Computer Science (SEECS), National University of Sciences and \\ Technology (NUST), Islamabad, Pakistan \\ ${ }^{b}$ Faculty of Science Engineering and Computing, Kingston University London, United Kingdom

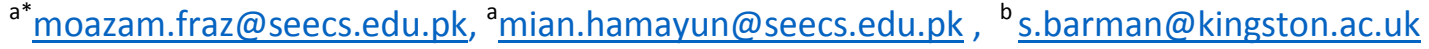

\section{Abstract}

Diabetic Retinopathy (DR) is the one among other main reasons of blindness in the adult population. Early discovery of DR through screening programs and successive treatment is critical in order to avoid visual blindness. The early signs of DR as manifested in retinal images include micro-aneurysms, hemorrhages and exudates. In this paper, we have presented an ensemble classifier of bootstrapped decision trees for multiscale localization and segmentation of exudates in retinal fundus images. The candidate exudates are extracted at fine grain and coarse grain levels using morphological reconstruction and Gabor filter respectively. The contextual cues are applied to the candidate exudates, which greatly reduces false positives in exudate segmentation. Several region based features are computed from candidate regions to train the ensemble classifier for classification of pixel as exudate and non-exudate region. The method has been evaluated on four publically available databases; DIARETDB1, e-Ophtha EX, HEI-MED and Messidor. The method has achieved the segmentation accuracy as $(0.8772,0.8925,0.9577$, and 0.9836$)$ and area under ROC as $(0.9310,0.9403,0.9842$, and 0.9961$)$ for each of the dataset respectively. The algorithm appears to be an efficient tool for automated detection of exudates in large population based DR screening programs, due to the attained accuracy, robustness, simplicity and speed.

Keywords: Medical Image Analysis; Feature Extraction; Ensemble Classification; Exudate Segmentation; Machine Learning; Diabetic Retinopathy

\section{Introduction}

Diabetes is a major chronic disease faced by the world population today and it is characterized by high levels of glucose in the blood. Diabetes manifests itself, when lack of insulin or insufficient action of insulin takes place in human metabolism, which leads to high blood glucose levels and is known as Hyperglycemia. Diabetes can damage small as well as large blood vessels, nerve cells, and resultantly cause damage to vital organs including brain, heart, kidneys and eyes. Damage to eyes primarily includes retinal complications collectively known as Diabetic Retinopathy (DR). The damage to retinal vasculature can lead to progressive retinal damage and result in partial vision loss or complete blindness [1]. DR is the most common reason for new cases of blindness among adult population[2]. The problem is increasing in scale, with diabetes identified as a significant growing global public health problem.

Clinicians commonly use retinal images for the screening of epidemic eye diseases like retinal edema and DR. The increasing pervasiveness of diabetes and low number of clinical specialists, increases the need for automatic methods to help reduce the work load on physicians [3]. 
Exudates are one of the earliest signs of DR and are formed by leakage of fluid from injured/damaged retinal blood vessels. The fluid has high content of proteins, white blood cells and cellular debris and appears as bright yellowish patches in the retinal images. This paper focuses on the detection of exudates, which are one of the main clinical symptoms of the presence of DR and Macular Edema. Due to their high contrast values, the exudates can be discerned more effectively from the retinal background in comparison with blood vessels and other anatomical structures. The leaked fluid can flow freely without restraint in the ocular fundus, therefore the exudates can form various irregular shapes and sizes. The noise, uneven illumination and contrast variations in images due to varying conditions during image capture, in addition to variability in shape, size and intensity add to the challenges of automated exudate detection from retinal images.

\section{Related Work}

There are a number of methodologies available in the literature for exudates detection in retinal fundus images [4]. Generally, the exudate detection approaches can be sub-divided into two main groups.

The first group contains methods based on mathematical morphology. Walter et al. [5] applied morphological closing for blood vessel elimination, followed by calculation of local standard deviation and thresholding to locate the candidate exudate regions. These techniques apply the morphological reconstruction to locate the exudate boundaries. Sopharak et al. [6] employed optimally adjusted mathematical morphology followed by morphological reconstruction to detect exudates in non-dilated retinal images. A coarse level segmentation of exudates was carried out by Jaafar et al. [7] using local variation calculation of image pixels, in order to outline the candidate boundaries. The adaptive thresholding results that have been obtained using the coarse level segmentation are further refined using a morphological operation. Welfer et al. [8] used the contrast enhanced $L$ channel of $L^{*} U^{*} V^{*}$ color spaces to apply morphological operations as well as $\mathrm{H}$-maxima transform for exudate detection. An Atlas based technique is proposed by Ali et al. [9]. Harangi et al. [10] have proposed an active contour-based region-wise method for identifying exudates. Most of these methods are prone to false positives near the vascular arch.

The second group is composed of machine learning based methods. These methods usually start with image normalization, followed by optic disc localization and removal. Then, the set of candidate regions, i.e. the structures similar to exudates are identified, followed by feature-set computations for each candidate region and classification for exudate regions. Different types of features have been suggested in literature. Pixel-wise features, which include pixel intensity, local variance, edge detection responses from sobel, Gaussian, difference of Gaussian and Gabor operators and wavelets [11]. Naive Bayes and fuzzy C-means have been used as classifiers in these cases. The pixel-wise features are supplemented with the addition of features like area, length or perimeter of candidate exudate regions [12]. This high dimensionality feature vector is computationally intensive to formulate. Acharya et al. [13] have used a Support Vector Machine (SVM) classifier fed with the non-linear feature vector computed from higher order spectra. Garcia et al. [14] proposed a classification methodology using multilayer perceptron, radial basis function and SVM classifier. Akram et al. [15] used a hybrid classifier comprising of a Gaussian mixture model (GMM) and SVM for improved exudates detection. The combination of GMM and SVM is also computationally demanding. Zhang et al. [16] has employed image normalization and de-noising followed by mathematical morphology to compute the feature vector, which is used to train Random Forest classifier for exudates detection. Pereira et al. [17] have used Ant colony optimization 
after identifying candidate exudate regions using connected component analysis. A method that includes features set based on color and trains the classifier using automatic lesion segmentation and wavelet decomposition has been introduced by Giancardo et al. [18]. Sánchez et al. [19] proposed a methodology which utilizes contextual information to improve lesion detection in medical images, resulted in significant gain in classification accuracy of pathologies.

In this paper, we present a methodology for exudates localization, detection and segmentation, which uses bootstrapped (bagged) decision trees based ensemble classifier. We combine the morphological operations, Gabor filter responses, contextual information obtained from retinal pathologies and machine learning techniques within a single unified framework. Most of the computer assisted systems use local information only to classify the candidate lesions, and do not consider the global image information. The proposed method takes into account the global image information as well as the relationship of candidate lesions with their neighboring anatomical structures within the retinal images. We have employed the spatial relationship between candidate exudates and surrounding anatomical structures (vascular arch, macula and optic disc) within the retinal image to improve the candidate exudate localization and the computation of quantifiable feature vector. The use of heuristic approach in feature vector generation has proved itself to be very beneficial in removing the artefacts and lesions that cause false exudate detection.

Another key contribution of the proposed technique is the use of ensemble classifier of bootstrapped decision trees for the classification of candidate regions into exudates and non-exudates. The ensemble classifier uses a feature vector obtained from Gabor filter responses, morphological reconstruction and top-hat filter application. The feature vector is a set of nine features that are used to characterize a given region as exudate or non-exudate region. The bootstrapped decision trees based ensemble, is a classic ensemble classifier that has been extensively applied in numerous image analysis application areas. To the best of our knowledge, the ensemble classifier has not been broadly employed for exudates segmentation in retinal funds images. Additional significant features of our bootstrapped ensemble classifier based exudate detection method include high classification accuracy, feature set computation and the conduct of training phase without requiring test data, which provides appreciable computational efficiency. The proposed algorithm is computationally fast during the training phase in addition to classification, as it needs fewer samples for training as compared to other supervised algorithms available in literature. The proposed algorithm has been evaluated for classification accuracy and robustness on four public image databases namely DIARETDB1, Messidor, e-Ophtha EX and HEIMED. Last but not the least, and to the best of our knowledge, this algorithm is the first one to be evaluated simultaneously on these four datasets.

This paper is organized as follows: Section 2 summarizes the exudate segmentation methodology, the feature vector formation and the ensemble classification approach. In section 3, the experimental evaluation, quantitative performance measures, and the robustness of the methodology are discussed in detail. We conclude this paper in section 4 and provide insights into future directions.

\section{The Methodology}

The exudates and other anatomical structures that are commonly found in retinal images are illustrated in Fig. 1 


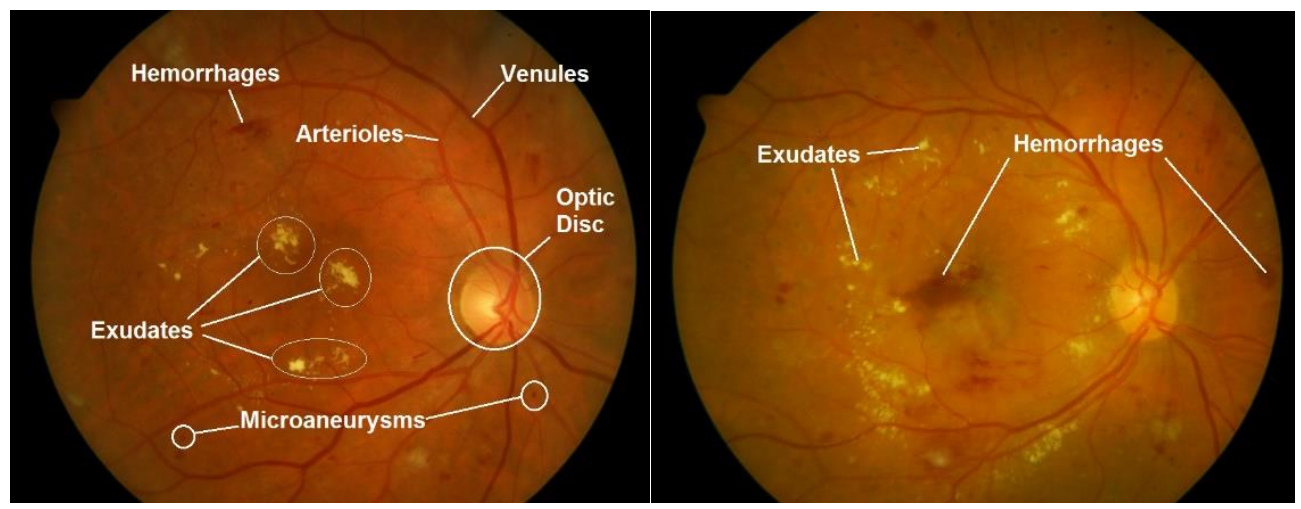

Fig. 1: Exudates and other anatomical structures in retinal images

Exudates show highest contrast with the background in the green channel of an RGB image, thus we have used the green channel of RGB images for exudates detection and segmentation. The green channel images are preprocessed for shade correction and normalization of uneven illumination. A series of image processing techniques are applied to get the candidate exudate regions. These techniques include morphological closing, histogram normalization, Gabor filter application, adaptive thresholding, morphological reconstruction and top-hat filtering.

A feature vector, consisting of nine features, has been designed to identify the candidate exudate regions and to compute the quantifiable measure for each candidate region. The ensemble classifier of bagged decision trees is employed to classify the candidate regions into exudates and non-exudates. The contextual information is used to remove regions in the retinal images that cause false exudate detection. These regions include the Optic Disc (OD), the main vascular arch area and the artefacts on the boundary of retinal images. The flowchart given in Fig. 2 illustrates different steps of proposed methodology for exudates detection.

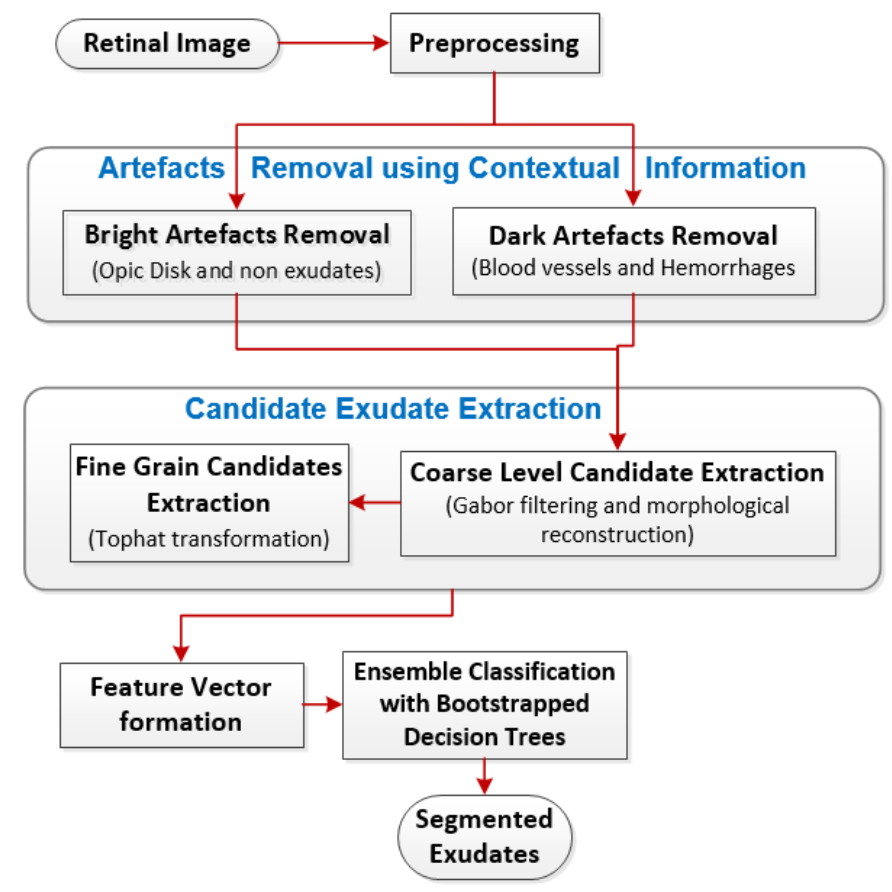

Fig. 2: Segmentation of Exudates in Retinal Images 


\subsection{Preprocessing}

The green channel of an RGB image gives maximum contrast between exudates and the neighboring regions [20]. The shade variability and contrast variations in retinal images largely contribute to false identification of candidate exudate regions in retinal images. Therefore, the green channel of RGB images is processed for normalization of contrast and luminosity. A variety of algorithms for contrast and luminosity normalization in retinal images are available in literature, and these methodologies are either based on subtracting the estimated background from the original image [21] or on dividing the later by the former $[22,23]$. However, experiments [21] show that the results of both methods are similar with no appreciable advantage of one over the other. We have used the subtractive method as it has been reported in [21]. Firstly, the background values are estimated and later the difference between the estimated background and the green channel is computed to produce the normalized image. The estimation of retinal image background, denoted by " $\mathrm{lbg}_{\mathrm{bg}}$ " is computed by the filtering the green channel image with a large arithmetic mean kernel. The filter kernel size is not a crucial parameter, for the reason that its size should be such that the resultant blurred image does not contain any visible anatomical structure. In this work, we have used an $89 \times 89$ pixel kernel. The difference between the estimated background " $I_{b g}$ " and the morphologically opened image " $I_{\text {open }}$ " is calculated on pixel basis. Thus the background normalized image " $I_{\text {norm }}$ " is obtained using:

$$
I_{\text {norm }}(x, y)=I_{\text {open }}(x, y)-I_{b g}(x, y)
$$

Due to different illumination conditions in the acquisition process, there may be significant intensity variations between images. These intensity variations make it hard to use a best possible technique for all of the images, thus shade corrections were necessary and have been applied. A global linear transformation function is applied to modify the pixel intensities such that the whole range of possible gray-levels ( [0-255], referred to 8-bit images) is covered.

$$
\begin{aligned}
& I_{S C}(x, y)=\left\{\begin{array}{cc}
0, & \text { if } I_{\text {norm }}(x, y)<0 \\
255, & \text { if } I_{\text {norm }}(x, y)>255 \\
I_{\text {adjusted }}(x, y) & \text { otherwise }
\end{array}\right. \\
& I_{\text {adjusted }}(x, y)=I_{\text {norm }}(x, y)+128-g l_{\text {max_pixels }}
\end{aligned}
$$

where $I_{S C}(x, y)$ is the shade corrected image, $I_{\text {norm }}(x, y)$ is the background normalized image, $\mathrm{gl}_{\text {max _pixels }}$ is the grey-level value of the highest pixel occurrence in the background normalized image $I_{\text {norm }}(x, y)$. The pixels with this intensity value $\mathrm{gl}_{\text {max }}$ pixels corresponds to the retinal background pixels. the background pixels [24]. These background pixels are set to 128 (for 8-bit grayscale image) hence the pixels with different illumination condition standardize their intensity around this value. The global transformation function illustrated in eq. (3) normalizes or shade corrects the image, which can be observed in Fig. 3. 


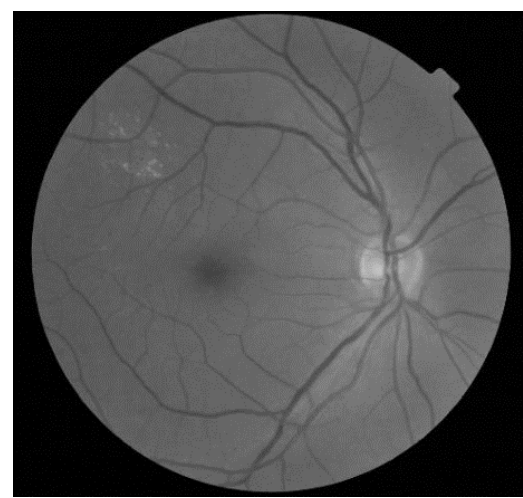

(a)

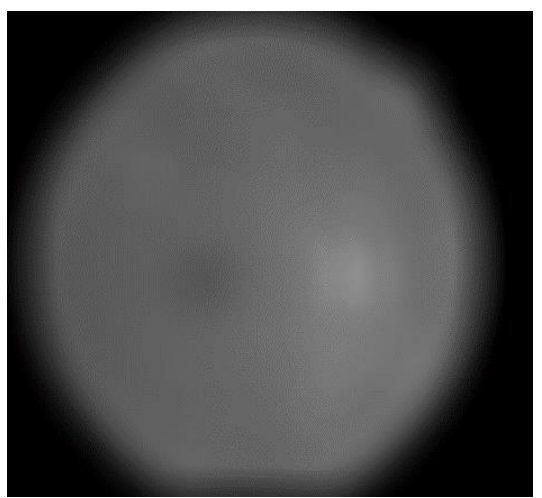

(b)

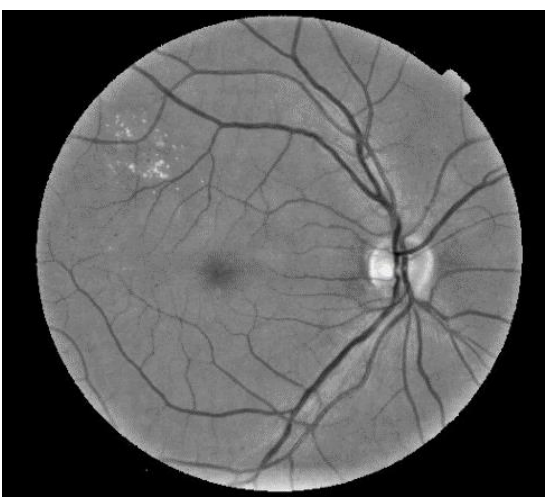

(c)

Fig. 3: Luminosity and Contrast Normalization: (a) Green Channel Image (b) Estimated Background Image ( $\left.I_{b g}\right)(c)$ Normalized Image

\subsection{Artefacts Removal}

There are several bright and dark artefacts in the retinal images, which can be erroneously identified as a candidate exudate. The bright artefacts include the Optical Disc (OD) and the area along the main vascular arch. The dark artefacts are the retinal blood vessels, hemorrhages and microaneurysms. The variability in contrast of the aforementioned artefacts with respect to the neighboring regions causes false detection of candidate regions and is a potential cause of poor performance of the exudate detection algorithms.

\subsubsection{Bright Artefacts Removal}

The OD and connected blood vessels in the main vascular arch area are the main cause of false exudates detection. Generally, these regions have a significant contrast with respect to their neighboring regions, thus they appear similar to exudates. From the contextual information of the retinal images, we have observed that there are almost always no exudates in the areas containing the optic disk and the vascular arch area [1]. Accurate OD detection, identification of main vascular arch area and removal of candidate exudates from this region in retinal images, greatly enhances the system accuracy. An efficient OD localization and boundary extraction methodology has been proposed in our research effort [25], and it gives $100 \%$ accuracy for OD detection in our dataset. After the detection of OD, a parabolic curve fitting is performed for the approximation of main vascular arch area [26]. The OD location and the approximation of main vascular arch by parabolic curve fitting on a retinal image are shown in Fig. 4. The candidate exudates from these regions are later removed. 


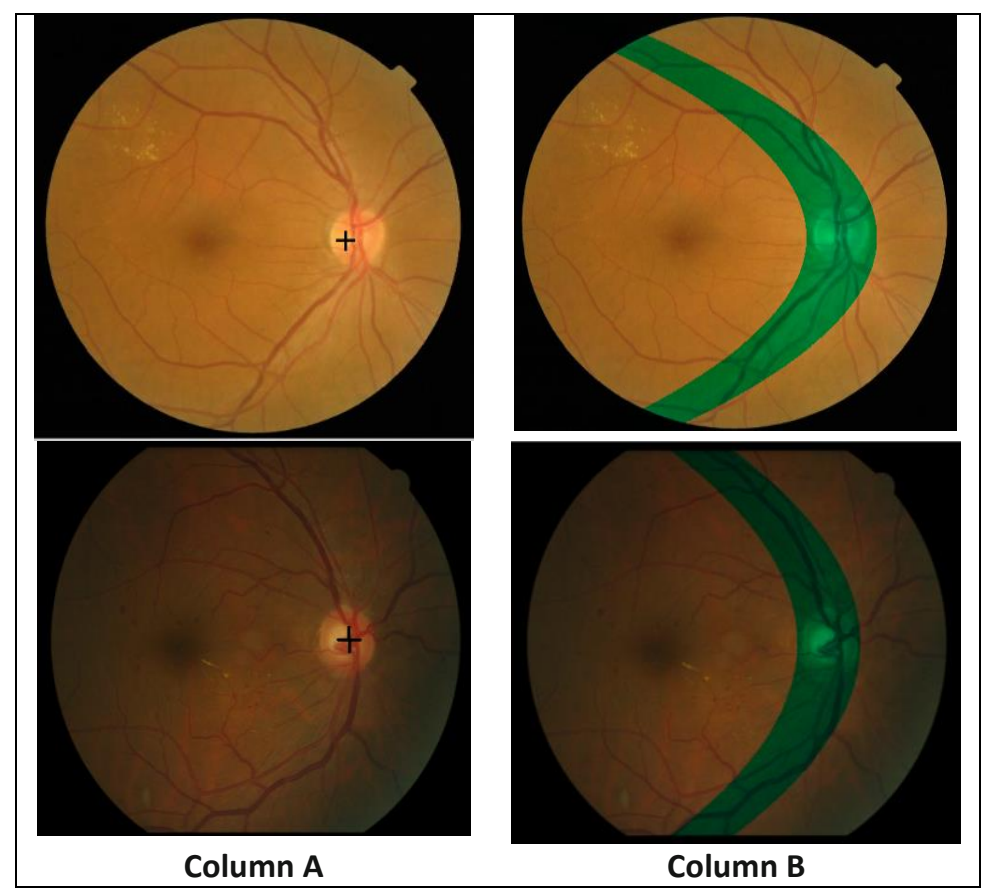

Fig. 4: Column A) OD Detected in Retinal Images; Column B) Parabolic Curve Fitting applied for bright artefacts removal

\subsubsection{Dark Artefacts Removal}

Vessels and other dark artefacts cause false exudate detection due to their contrasting nature as compared to the neighboring regions. Thus these dark artefacts should be removed. In order to eliminate the vessels and unwanted dark regions from the retinal fundus images, we use the image inpainting technique by applying morphological closing [27].

Mathematical morphology [27] is revealed to be a very useful technique for quantifying pathologies in retinal images [28]. Morphological closing operation removes most of the unwanted dark artefacts. The "Octagon" structuring element is used for closing operation. The size of the octagon has been chosen according to the width of the largest vessel in the image, similar to the approach proposed in [24]. This gives a suitable approximation of vessel width ' $d$ ' for all the datasets under consideration. The removal of the retinal vasculature is shown in Fig. 5. 


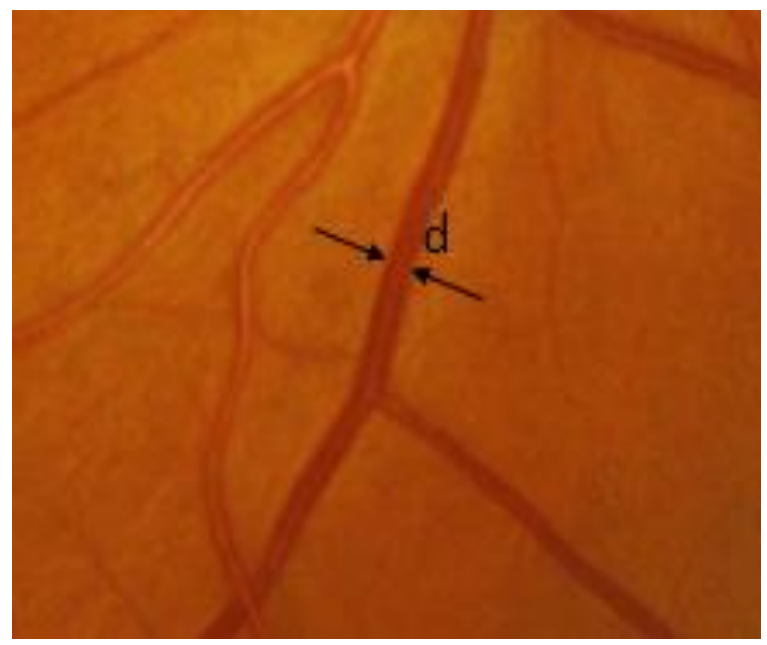

(a)

Fig. 5 : Dark artefact removal

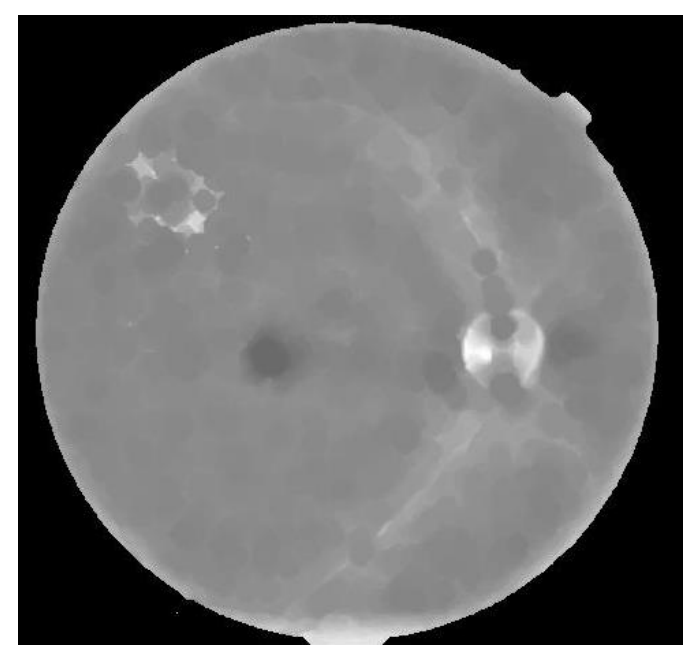

(b) retinal image

\subsection{Candidate Extraction}

The exudates appear in variable sizes and shapes, with regions ranging from a few pixels to considerably large areas. Most of the exudate detection algorithms available in the literature do not cater for the variability in exudate sizes and treat them similarly. We have employed a two pronged strategy for candidate exudates identification. After the removal of artefacts, the retinal images are processed using a series of image processing techniques focusing on identification of large and small candidate regions independently.

\subsubsection{Coarse Grain Exudate Candidates Extraction}

The preprocessed image is used for the large candidate exudates identification at coarse grain level. Adaptive contrast enhancement is applied to the preprocessed (vessel removed) images, in order to improve the contrast between potential exudates and their neighboring regions. A sample result of adaptive contrast enhancement is illustrated in Fig. 6 (a). A Gabor filter is applied to the contrast enhanced images to find the boundaries of candidate exudates. In general, the Gabor filter is employed for multi-directional and multi-scale detection of edges. The Gabor filter has the property that it can be easily tuned to specific frequencies, directions and scale, thus possessing the capability of low level feature extraction and background noise suppression. The Gabor filter kernel can be illustrated as the product of a complex sinusoid and a Gaussian kernel, which is mathematically expressed as,

$$
g(x, y)=\exp \left[-\frac{1}{2}\left(\frac{x^{\prime 2}+\gamma y^{\prime 2}}{2 \sigma^{2}}\right)\right] \exp \left[i\left(2 \pi \frac{x^{\prime}}{\lambda}+\psi\right)\right]
$$

Where, $x^{\prime}=x \cdot \cos \theta+y \cdot \sin \theta, y^{\prime}=-x \cdot \sin \theta+y \cdot \cos \theta ; \theta$ represents the orientation, $\psi$ characterize the phase offset, $\sigma$ shows the Gaussian envelop scale and $\lambda$ illustrates the spatial aspect ratio. The Gabor filter is implemented as described in [29]. The Gabor filter is constructed at two scales (numeric values 2 and 5), on two orientations (pi/4 and pi/2) and the set of frequencies are [0.5 and 2]. 


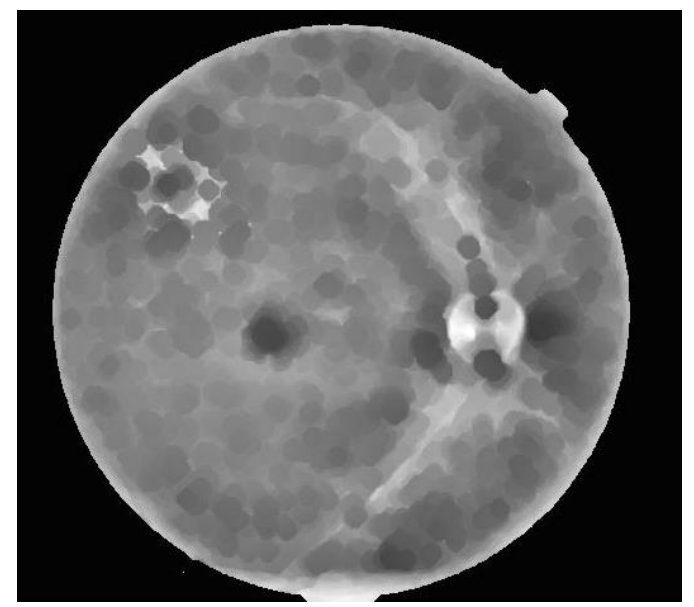

(a)

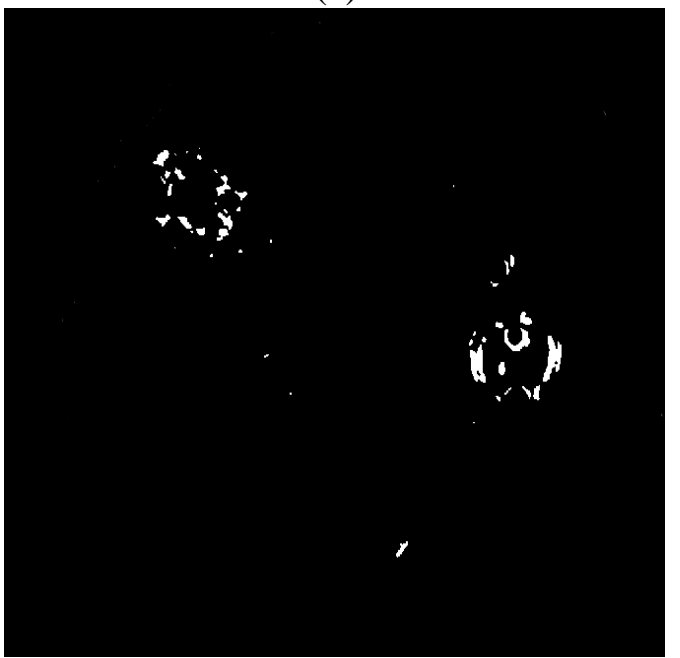

(c)

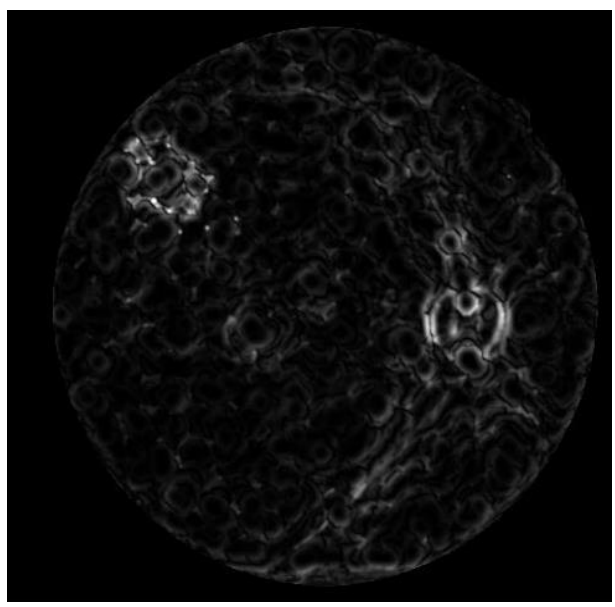

(b)

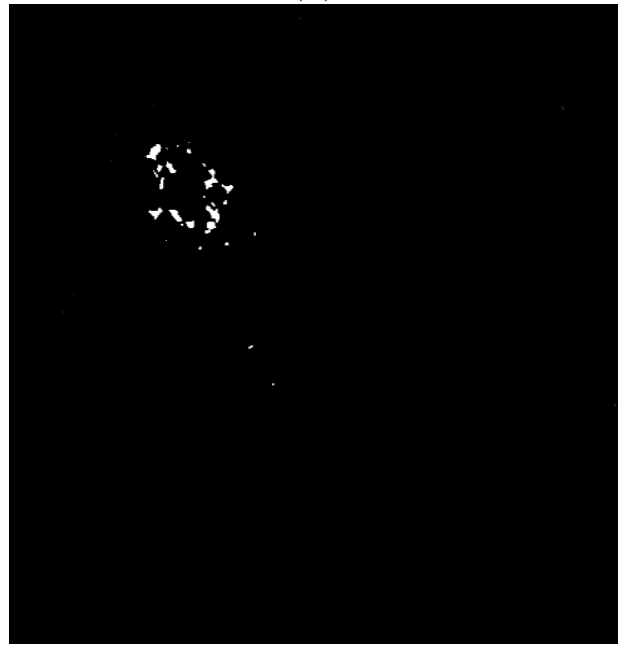

(d)

Fig. 6: (a) Contrast Enhanced Image (b) Gabor Filter Response (c) Result of morphological reconstruction (d) Candidate exudates removed based on contextual information

The Gabor Filter Response (GFR) image is illustrated in Fig. 6 (b). It has been observed that the shape of large exudates is not very accurate if Otsu's adaptive thresholding [30] has been applied to the GFR image. Therefore, in order to attain a better approximation of the shape of large candidate exudates, a hysteresis thresholding based morphological reconstruction is applied to the GFR image. The details of this procedure have been reported by the authors elsewhere $[31,32]$. In this technique, the GFR image which is actually a grey level image has been thresholded for two ranges of pixel intensity values, thus employing a bi-threshold procedure. In the first instance, the GFR image is thresholded by a narrow range (T1), which results in the segmentation of high confidence exudate pixels as well as many false negative responses. This image is labelled as the "marker image". After wards, a wide range threshold (T2) is applied to the GFR image to produce a "mask image". These threshold values (T1 and T2) are obtained from the intensity histogram of the non-null pixels of the GFR image. The T1 or T2 are computed as the highest pixel intensity value such that pixel count with the intensities above this limit (T1 or T2) is greater or equal to a predefined percentage in the histogram. This percentage value is empirically selected for $\mathrm{T} 1$ and $\mathrm{T} 2$ as $90 \%$ and $95 \%$ respectively. The obtained marker and mask images are used for morphological reconstruction [31]. 
In general, from the contextual information of retinal pathology, it is known that the exudates are not present near the OD and main vascular arch area. The regions of the OD and main vascular arch in the retinal image are approximated as explained in section 3.2.1, and illustrated in Fig. 4. Therefore, the candidate regions in the aforementioned area of the morphologically reconstructed image are removed in order to reduce the false positive detection of candidate exudates, as illustrated in Fig. 6 (d).

\subsubsection{Fine Grain Exudate Candidates Extraction}

The fine grain candidate exudate regions are extracted by applying a morphological top-hat operation using the octagon shaped structuring element followed by adaptive thresholding using Otsu's algorithm [30].

The morphological erosion followed by the dilation procedure which is also known as the morphological opening operator $(\mathrm{MOO})$ removes those objects from the image which are smaller in size than the Structuring Element (SE) used by the operator [27]. The objects which are removed by the MOO are enhanced in contrast if the morphologically opened image is subtracted from the original image. Without loss of generality, we assume that the exudates are irregular shaped bright patterns on the dark background. Due to their irregular roundish shapes, the MOO with the octagon shaped SE are utilized for contrast enhancement of candidate exudates in the retinal fundus images. MOO using an octagon shaped SE will remove the candidate exudates when its size large enough such that it cannot be enclosed within the candidate exudate region. The size of octagon shaped SE is chosen to be approximated to the half radius of the maximum vessel width in the retinal images, a similar approach has been used by [24]. The morphological top-hat operation is illustrated in (5) where $I_{F G}$ represents the fine grain candidate exudate image obtained by the top-hat transformation, $l$ is the image to be processed and $S_{e}$ is the octagon shaped structuring elements for morphological opening " $O$ ".

$$
\begin{gathered}
I_{\mathrm{FG}}=I-\left(\begin{array}{lll}
\text { I } & \text { o } & S_{\mathrm{e}}
\end{array}\right) \\
I_{\text {cand_exudates }}=\operatorname{supremum}\left(I_{\mathrm{FG}}, I_{\mathrm{CG}}\right)
\end{gathered}
$$

In order to combine the candidate exudate regions at the coarse grain and fine grain levels, the supremum of the images obtained at each of the scales is taken as is illustrated in (6). $I_{\text {cand_exudates }}$ is the image containing candidate exudate regions, $I_{\mathrm{FG}}$ and $I_{\mathrm{CG}}$ are the candidate exudate images at fine grain and coarse grain levels, respectively. The results of the fine grain exudate extraction and supremum operator are presented in Fig. 7.

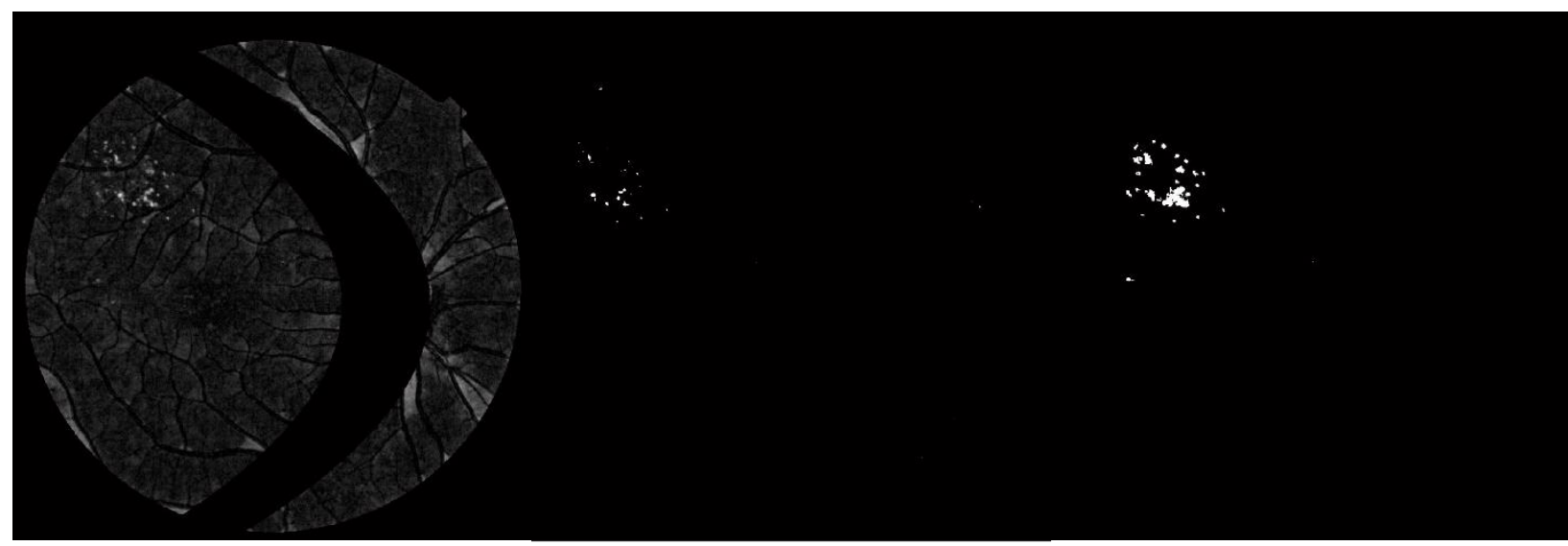

(a)

(b)

(c) 
Fig. 7 : a) Top-hat filtered image b) Fine grain candidate exudate regions c) Candidate Exudate Regions obtained by Supremum Operator

\subsection{Feature Vector}

Each candidate region in the retinal image is characterized by a feature vector in 9-D feature space, in order to distinguish between the candidate regions as exudates and non exudates

$$
F v(x, y)=\left[f_{1}(x, y), . f_{2}(x, y) \ldots \ldots f_{9}(x, y)\right]
$$

A classification technique allocates one of the classes from $C_{\text {EXUdATE }}$ (exudate) or $\mathrm{C}_{\text {NON-EXUdATE }}$ (nonexudate) to each candidate region when its representation in feature space $F v(x, y)$ becomes known.

Clinical experts have been asked how they identify the exudate regions in the retinal images, so that the feature extraction process incorporates the cues used by the clinician's for exudate detection in retinal image. After deliberations, the size, color, texture and the shape are found to be the most significant features that are considered for exudate classification by ophthalmologists. We try to mimic a clinician's expertise by extracting relevant features from the candidate regions for differentiating between exudate pixels and non-exudate pixels. Each candidate exudate region is represented by a feature vector containing nine region based features. The chosen feature vector is explained in Table 1.

Table 1 : Feature Vector for Candidate Exudates

\begin{tabular}{|c|c|c|c|}
\hline S. No & $\begin{array}{l}\text { Feature } \\
\text { Name }\end{array}$ & Details & Mathematical Expression \\
\hline 1 & Area (A) & $\begin{array}{l}\text { The size of the candidate } \\
\text { region }\end{array}$ & Total pixel count in the candidate region \\
\hline 2 & $\begin{array}{l}\text { Compactness } \\
\text { (C) }\end{array}$ & $\begin{array}{l}\text { Magnitude of density of the } \\
\text { candidate region }\end{array}$ & $C=\frac{\text { perimeter }^{\wedge} 2}{4 * \text { pi } * \text { area }}$ \\
\hline 3 & $\begin{array}{l}\text { Mean green } \\
\text { channel } \\
\text { intensity }\left(\mu_{\mathrm{g}}\right) \\
\end{array}$ & $\begin{array}{l}\text { Average green channel } \\
\text { intensity of the candidate } \\
\text { region }\end{array}$ & $\left(\mu_{g}\right)=\frac{\text { Sum of Green channel intensity of pixels in region }}{\text { Number of pixels in the region }}$ \\
\hline 4 & $\begin{array}{l}\text { Mean Hue } \\
\text { Channel } \\
\text { Intensity in } \\
\text { HSV color } \\
\text { space } \\
\left(\mu_{\mathrm{h}}\right)\end{array}$ & $\begin{array}{l}\text { Average Hue channel intensity } \\
\text { of the candidate region in HSV } \\
\text { color space. It is an attribute of } \\
\text { visual sensation according to } \\
\text { which an area appears to be } \\
\text { similar to the perceived color }\end{array}$ & $\left(\mu_{h}\right)=\frac{\text { Sum of hue value of pixels in region }}{\text { Number of pixels in the region }}$ \\
\hline 5 & $\begin{array}{l}\text { Mean } \\
\text { Saturation in } \\
\text { HSV color } \\
\text { space } \\
\left(\mu_{s}\right)\end{array}$ & $\begin{array}{l}\text { Average "S" channel intensity } \\
\text { of the candidate region in HSV } \\
\text { color space. It depicts the } \\
\text { strength or purity of the color } \\
\text { of the candidate region. }\end{array}$ & $\left(\mu_{S}\right)=\frac{\text { Sum of saturation value of pixels in region }}{\text { Number of pixels in the region }}$ \\
\hline \multirow[b]{2}{*}{6} & \multirow{2}{*}{$\begin{array}{l}\text { Mean "V" } \\
\text { value of HSV } \\
\text { color space } \\
\left(\mu_{\mathrm{v}}\right), \text { which is } \\
\text { the Lightness } \\
\text { component }\end{array}$} & \multirow{2}{*}{$\begin{array}{l}\text { Average of the "V" channel } \\
\text { value of the candidate region } \\
\text { in HSV color space. It is the } \\
\text { representation of brightness of } \\
\text { color in the candidate region. }\end{array}$} & \multirow{2}{*}{$\left(\mu_{V}\right)=\frac{\text { Sum of the Lightenss value of pixels in region }}{\text { Number of pixels in the region }}$} \\
\hline & & & \\
\hline \multirow{2}{*}{7} & \multirow{2}{*}{$\begin{array}{l}\text { Mean gradient } \\
\text { magnitude } \\
\left(\mu_{\text {grad } \mathrm{mag}}\right)\end{array}$} & \multirow{2}{*}{$\begin{array}{l}\text { Directional change in intensity } \\
\text { of the candidate region pixels. }\end{array}$} & \multirow{2}{*}{$\mu_{\text {grad_mag }}=\frac{\sum(\text { gradient img pixel intensity in region })}{\text { No. of pixels in the region }}$} \\
\hline & & & \\
\hline 8 & Entropy (Ent) & $\begin{array}{l}\text { A statistical metric to measure } \\
\text { the randomness. Entropy } \\
\text { characterize the texture of } \\
\text { candidate exudate region }\end{array}$ & $E n t=-\sum_{i} P\left(x_{i}\right) \log _{2} P\left(x_{i}\right)$ \\
\hline 9 & Energy (Enr) & $\begin{array}{l}\text { Measure of how the spectral } \\
\text { density varies with frequency. }\end{array}$ & $\begin{array}{c}\text { Enr = sum of intensity squares of all pixel values in green } \\
\text { channel of RGB }\end{array}$ \\
\hline
\end{tabular}




\subsection{Ensemble Classification}

Ensemble classification is the methodology for generation and combination of multiple classifiers with the aim to find an optimal solution of a particular machine learning problem [33]. Ensemble classification is used for improving the prediction performance of the classifiers in combination with the reduction in probability of false prediction. Ensemble classification uses the approach used in daily life, where people seek suggestions from different people about a problem's solution, weigh them and then decide in order to make a better and well-informed decision. The usage of multiple classifiers in the ensemble methods improve the predictive performance, by aggregating the outcomes of multiple weak learners in to a single ensemble classifier. In the proposed methodology, the decision trees are used weak learner and their outcome is combined with bagging also known as bootstrap aggregation. [34]. One of the key advantages of bagged ensemble classifiers is that their predictive performance can be tested by using Out-Of-Bag (OOB) training samples without supplying any test data.

\subsubsection{Bootstrap Aggregation}

The bootstrapped aggregation algorithm also known as bagging, proposed by Breiman's [34], is one of the earliest, most intuitive and simplest to implement ensemble based algorithm. In bagging, the component classifiers (the decision trees in this case) are trained on the bootstrap replicas of the training data. The bootstrapped training data is generated by random selection of $\mathrm{P}$ training samples out of $Q$, where $Q$ is the size of training set. The individual classifier's predictive outcome is strategically combined by majority voting. The ensemble classifier decision is the label chosen by majority of the weak learners.

Let us suppose that the original training set is " $\mathrm{T}$ ", the multiple sets of training data " $\mathrm{T}_{\mathrm{M}}$ " are formed by random sampling of training set " $\mathrm{T}$ " with replacement. " $\mathrm{M}$ " is the number of decision trees used in the ensemble. The random selection with replacement make sure that each training set " $\mathrm{T}_{\mathrm{M}}$ " contains not more that $67 \%$ of the original training samples. The implementation details of bagging algorithm as explained in [35] are shown as,

Given the original training set $D$, multiple sets of training data $D_{m}$ are created, where $\mathrm{m} \in\{1,2, \ldots \mathrm{N}\}$ by randomly sampling $D$ with replacement. $\mathrm{M}$ is the number of component classifiers used in the ensemble system. On average, each training set $D_{m}$ only contains two-thirds of the original samples. The bagging algorithm as explained in is illustrated below,

Bagging Algorithms Inputs

- Training data $T=\left(a_{1}, a_{2}, \ldots \ldots . ., a_{m}\right\}, a_{i} \varepsilon M \quad$, with correct labels $l_{i} \in \Omega=\left\{l_{1}, \ldots ., l_{C}\right\}$

- Decision Tree Learning algorithm DTLearn(),

- Integer $K$, specifying the training iterations.

Training the Classifier

Do $k=1, \ldots, k$

1) Create bootstrap sample with replacement $T_{m}$ by randomly drawing $m$ instances from $T$.

2) Call DTLearn() with training set $T_{m}$ and obtain the trained component classifier $C_{k}: Y \rightarrow \Omega$.

3) Add $C_{k}$ to the ensemble, $\boldsymbol{E}$.

End Do Loop 
Test: The Classifier - given the unlabelled instance " $\mathrm{z}$ "

1. The Ensemble classifier $E=\left\{C_{1}, C 2 \ldots . ., C_{B}\right\}$ is evaluated on test data " $Z$ "

2. The outcome given to class $l_{j}$ by the component classifier $C_{k}$ be

$$
v_{k, j}=\left\{\begin{array}{lr}
1, & \text { if } h_{k} \text { picks class } l_{j} \\
0, & \text { otherwise }
\end{array}\right.
$$

3. The total vote got by each class is obtained by majority voting as

$$
V_{j}=\sum_{k=1}^{K} v_{k, j}, \quad j=1, \ldots \ldots, C .
$$

4. The class that has obtained the highest total vote is chosen as the final label of test data

\subsubsection{Out-Of-Bag Classification Error}

The picking up of $X$ out of $Y$ training samples with replacement, leave behind on average $37 \%$ of the training samples for each weak learner, which is the decision tree in his case. These samples are known as "Out-Of-Bag" (OOB) observations on the training set, which are not used in the individual decision tree training. Thus, these observations can be used to estimate the predictive power of a classifier as well as the importance of each individual feature from the feature vector, in the decision-making process.

The OOB predicted outcomes are compared with the known outcomes of training samples in order to compute $\mathrm{OOB}$ classification error, which can be considered as the approximation of ensemble classification error. For estimating the importance of particular feature in prediction, the $\mathrm{OOB}$ data is randomly permutated across one variable at a time and resulted variance of $\mathrm{OOB}$ classification error is analyzed. The higher the value of OOB classification error, more significant the feature will be in the classification. In this way the approximation of classifier's predictive power and the importance of a particular feature in classification can be computed during the classifier training without using the test data. These capabilities make the ensemble a smart choice for classification.

Fig. 8 shows the plot of $\mathrm{OOB}$ classification error computed for the classifiers comprising of 100 decision trees, which have been trained with 5600, 10000 and 12000 samples. It can be seen in the graph that there will be insignificant increase in classification accuracy at the cost of processing time, if 40 or more decision trees are used to construct the ensemble classifier. Therefore, 40 decision trees have been chosen for constructing the ensemble classifiers. Moreover, it has been observed that the OOB classification error for the ensemble trained with 5600, 10000 and 12000 training samples, is approximately same. Therefore, the ensemble can be optimally trained using 5600 training samples thus further reducing the computational load. 


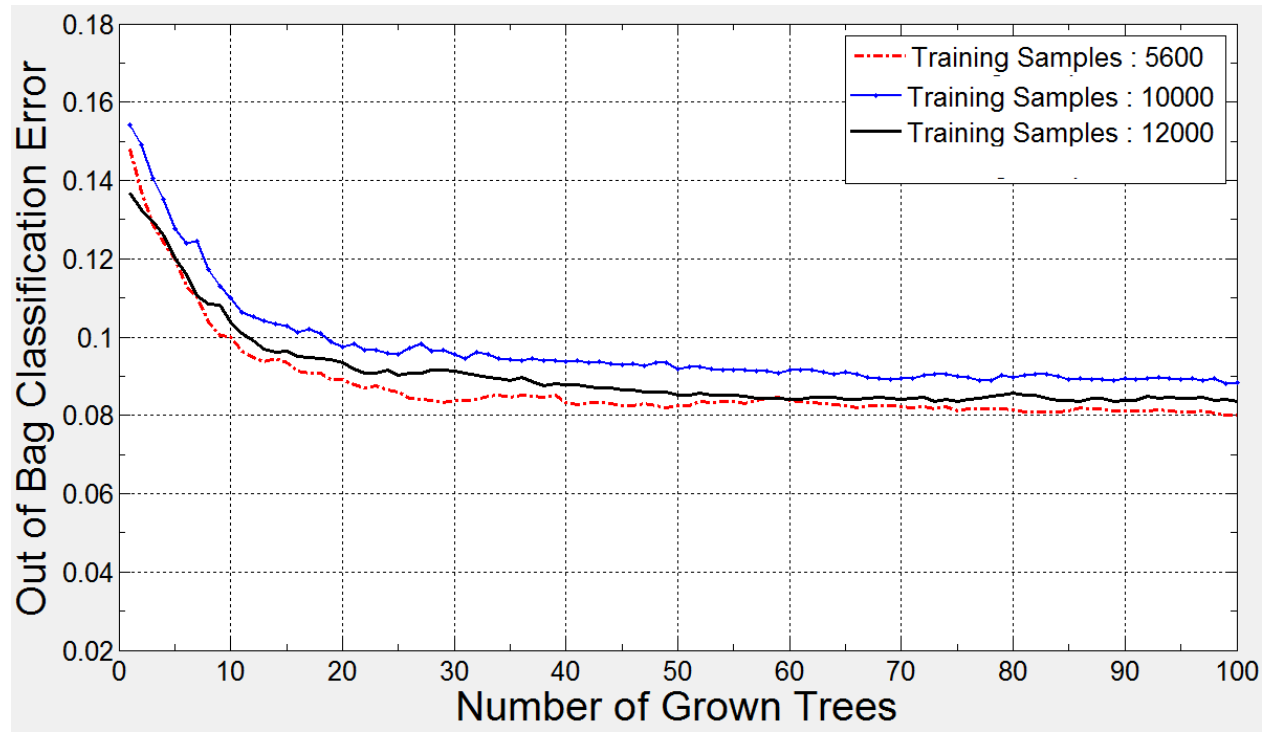

Fig. 8: OOB Classification Error for estimating the optimal number of Decision Trees and Training Samples

\subsection{Exudates Ground Truths Marking Tool}

To validate the exudates classification performed by the proposed algorithm, we need a reference standard or a ground truth for comparison purposes. The exudates in the retinal images should be manually marked by clinical experts. This ground truth will be used for classifier training and later on for calculating the performance measures of our classifier on a set of test retinal images.

Keeping in mind the above requirement, a software tool has been developed, in which a retinal fundus image can be loaded, magnified and zoomed to various levels. This tool allows the clinical experts to precisely mark the exudates in retinal images, even up to a pixel level using simple mouse clicks. The clinical experts can edit their marking as well. A snapshot of this tool is given in Fig. 9. 


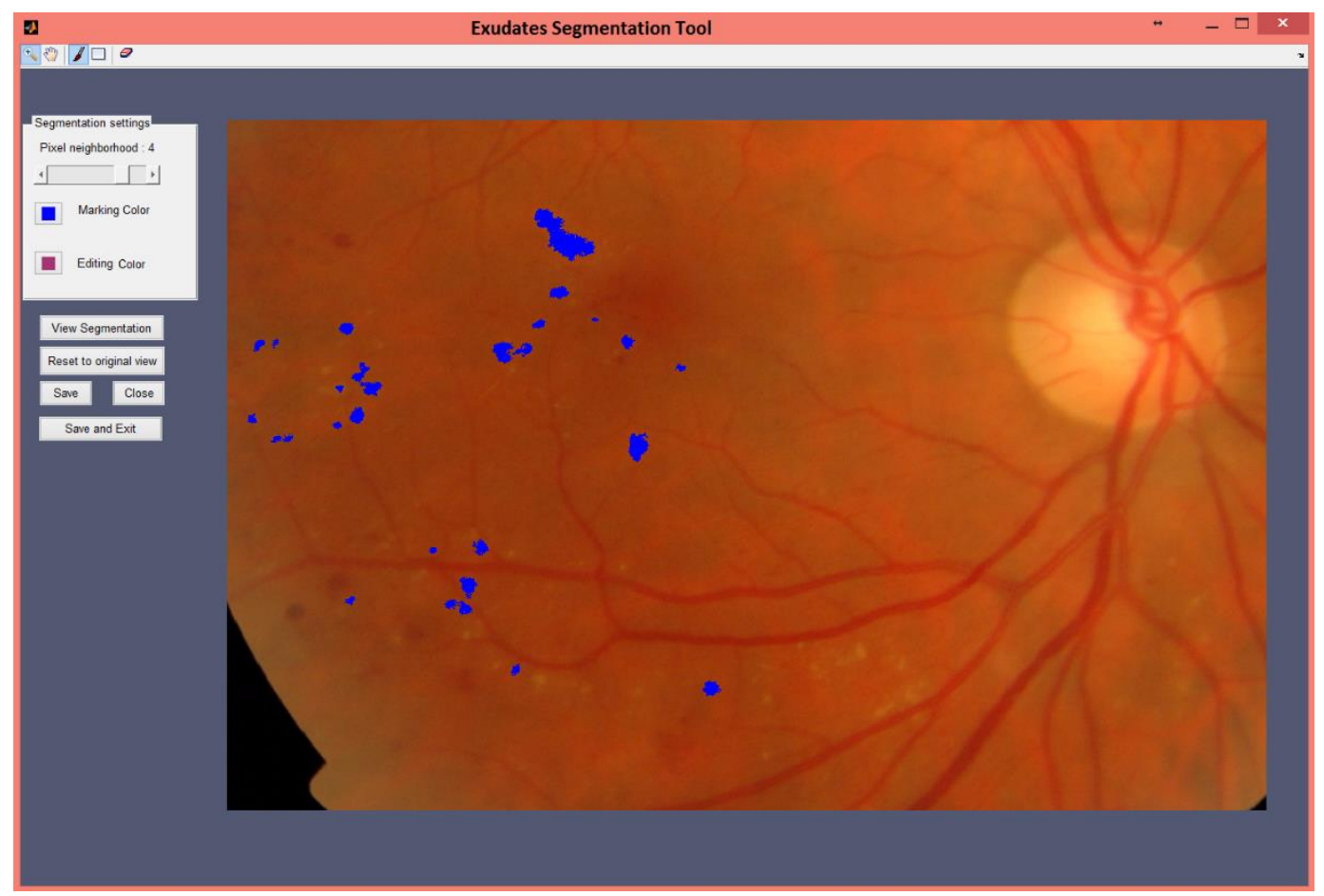

Fig. 9: Exudates Segmentation Tool

\section{Results and Discussion}

The exudates detection performance can be evaluated at two different levels; the pixel level validation and the image level validation. The prerequisite for the pixel level validation is the availability of manually segmented exudates created by clinical experts. For this purpose, we have created an interactive software tool for clinical experts. This tool provides the functionality of precise marking of exudates in the retinal images at pixel level (section 3.6). The retinal images were provided to our clinical collaborators i.e. the ophthalmologists at the Armed Forces Institute of Ophthalmology, Rawalpindi, Pakistan. Our clinical experts have manually created the exudates ground truths using our software tool. At the pixel level validation, the evaluation is performed by counting the correctly identified pixels in the retinal image as exudates and non-exudates, in comparison with the manually created ground truths. The performance metrics of Sensitivity, Specificity, Accuracy and Precision are computed by using the number of correctly identified exudate and non-exudate pixels. The details of our evaluation methodology are given in the following sub-sections.

\subsection{Materials}

The method is evaluated of four public retinal image datasets (DIARETDB1, e-Ophtha Ex, HEI-MED and Messidor). The exudates in the retinal images are manually marked by clinical experts using the software tool described in the section 3.6. The manually marked retinal images are considered as the reference standard and utilized for the evaluation of proposed methodology.

\subsubsection{DIARETDB1}

DIARETDB1 database [36] consists of 89 colored retinal images. The training set containing 26 images and the test set consists of 63 images. The ensemble classifier is trained using 3600 samples. 


\subsection{2 e-Ophtha EX}

The e-Ophtha EX database [37] consists of 82 colored retinal images. Approximately 2200 samples from 22 images are used for training and the test set is comprised of 60 images.

\subsubsection{HEI-MED}

HEI-MED database [38] consists of 169 colored retinal images. The training set is comprised of 39 images and a test set contains 110 images. The ensemble classifier is trained using 5600 sample observations.

\subsubsection{Messidor}

158 images have been taken from the Messidor retinal image-set [39] containing exudates and similar pathologies. The training set contains 50 images and a test set is comprised of 108 images. The ensemble classifier training is performed using 12000 sample observations.

\subsection{Performance Measures}

In the classification of candidate regions, the candidate image region is either classified as exudate or a non-exudate. There are four main events in classification of exudates. Two of them are classifications and the other two are misclassifications as shown in Table 2.

Table 2 : Exudate Classification

\begin{tabular}{|l|l|l|}
\hline & $\begin{array}{l}\text { Exudate pixels in manually } \\
\text { segmented image }\end{array}$ & $\begin{array}{l}\text { Non-Exudate pixels in } \\
\text { manually segmented image }\end{array}$ \\
\hline Exudate pixels detected by algorithm & True Positive (TP) & False Positive (FP) \\
\hline Non-exudate pixels detected by algorithm & False Negative (FN) & True Negative (TN) \\
\hline
\end{tabular}

Sensitivity (SN) is the ability of method to detect exudate pixels among candidate regions. Specificity (SP) measure shows the ability to detect non-exudate pixels among candidate regions. Accuracy (Acc) is calculated as the ratio between the count of accurately identified exudate pixels and the total pixel count in the candidate regions. The Positive Predicted Value (PPV) is considered as the probability that identified exudate pixel is true positive. The False Detection Rate (FDR) is taken as the probability that identified exudate is false positive. These performance parameters are listed in Table 3.

Table 3 : Performance Measures

\begin{tabular}{|l|l|}
\hline Performance Measure & \multicolumn{1}{c|}{ Mathematical Formula } \\
\hline SN & TP / ( FN + TP ) \\
\hline SP & TN / ( FP + TN ) \\
\hline ACC & (TN + TP) / (FP + TP + FN + TN ) \\
\hline PPV & TP / ( FP + TP ) \\
\hline FDR & $\mathrm{FP} /($ FP + TP ) \\
\hline
\end{tabular}

In our experiments, these performance parameters are calculated over all test images. Additionally, the algorithm performance is evaluated by using the Area Under Receiver Operating Characteristic (ROC) curve. An ROC curve is a graph of Sensitivity (SN) and the false positive fraction (1-SP).

\subsection{Exudate Classification Results}

Each candidate exudate region is characterized by a feature vector. The ensemble classifier assigns one of the classes $C_{\text {exudate }}$ (exudate) or $\mathrm{C}_{\text {Non_exudate }}$ (non-exudate) to each of the pixel in the candidate exudate region. This method designates one of the class label $C_{\text {EXUDATE }}$ or $C_{\text {NON_EXUdATE }}$ to each of the 
exudate region. The outcome of ensemble classifier is the confidence measure of each candidate region to be an exudate or non-exudate. The classifier generates a probability map for candidate exudate regions, where each value can be considered as the confidence measure for every pixel to be classified as an exudate or non-exudate region.

The probability map image is thresholded to produce a binary image in such a way that each pixel in the PM image is assigned with one of the class label ( $C_{\text {EXUDATE }}$ or $\left.C_{\text {NON_EXUDATE }}\right)$, depending upon its associated probability value. The assignment of binary values 1 and 0 to the class labels can be given mathematically as,

$$
I_{R E S}(x, y)=\left\{\begin{array}{c}
1\left(\equiv C_{\text {Exudate }}\right), \rho\left(C_{\text {Exudate }} \mid F v(x, y)\right) \geq T \\
0\left(\equiv C_{\text {Non_Exudate }}\right), \text { Otherwise }
\end{array}\right.
$$

Where $\rho\left(C_{\text {Exudate }} \mid F v(x, y)\right)$ is the probability of a pixel $(x, y)$ belongs to class $C_{\text {EXUDATE }}$ given the feature set $F v(x, y)$.

The binary image is generated for several threshold values and the respective accuracy is computed for each particular threshold value. The threshold value associated with best accurate is selected for each of the retinal image-set. Fig. 10 illustrates the graph of accuracy versus the threshold values $T$ on the probability map image, which are used for computing the $I_{R E S}$ as defined in (10), for each of the retinal image datasets.

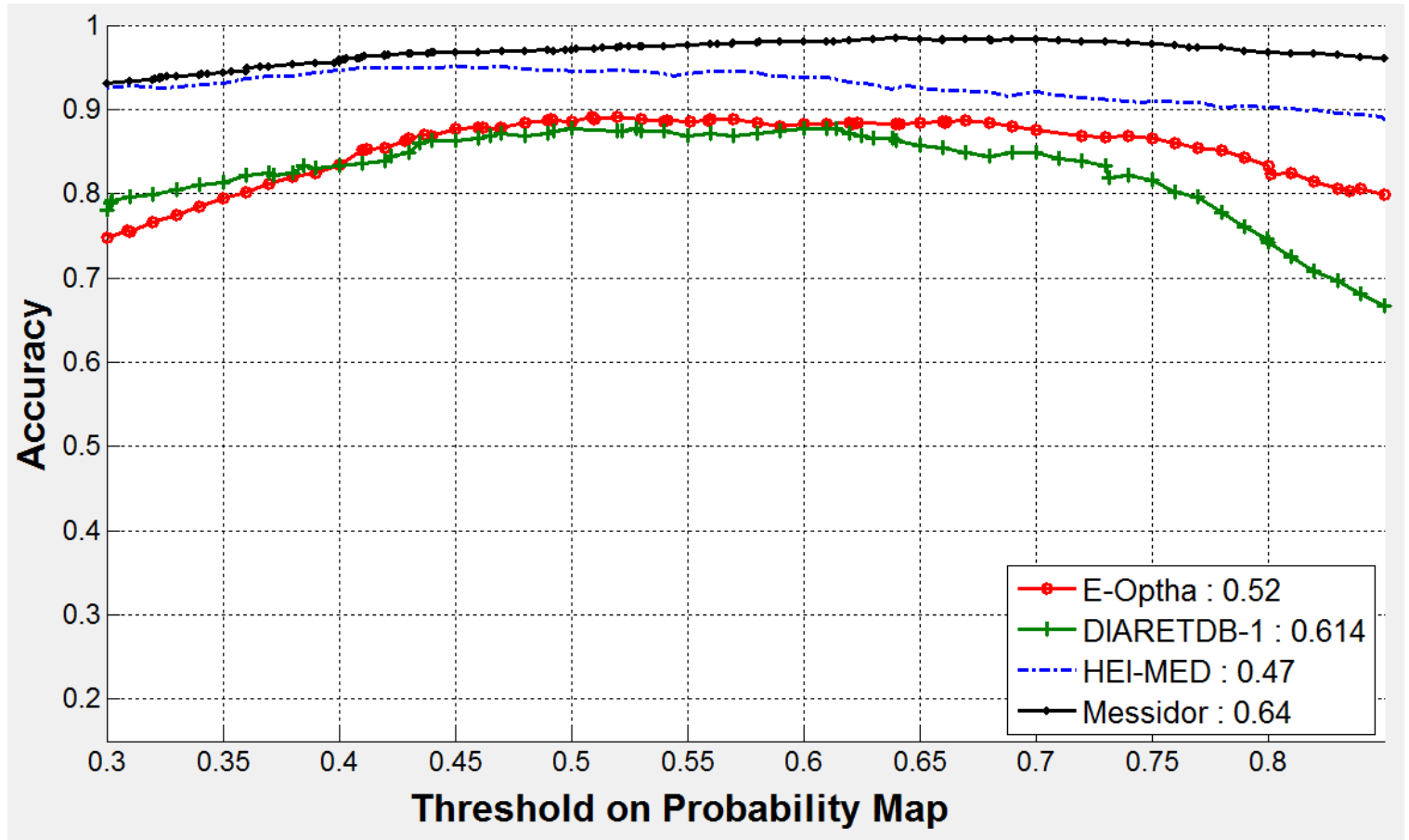

Fig. 10 : Method Accuracy vs. the optimal threshold for $C_{\text {EXUDATES }}$

The OOB observations are used to determine the optimal number of decision trees utilized to create the ensemble classifier, as well as the count of training samples for classifier training. We have created one ensemble classifier for each of the retinal images datasets, using 40 bootstrapped decision trees and trained it with 10000 training samples. The trained classifier is then employed for exudate detection on the DIARETDB1, e-Ophtha EX, HEI-MED and Messidor images. A vector of SN and SP is obtained for test 
data of respective image databases. The ROC is plotted for each database and the area under ROC is computed using these vectors, as shown in Fig. 11. The ROC values for the databases are summarized in Table 4.

Table 4 : Area under ROC for exudate classification in different retinal image datasets

\begin{tabular}{|l|l|}
\hline Database & Area under ROC \\
\hline DIARETDB1 & 0.9310 \\
\hline e-Ophtha EX & 0.9403 \\
\hline HEI-MED & 0.9842 \\
\hline Messidor & 0.9961 \\
\hline
\end{tabular}

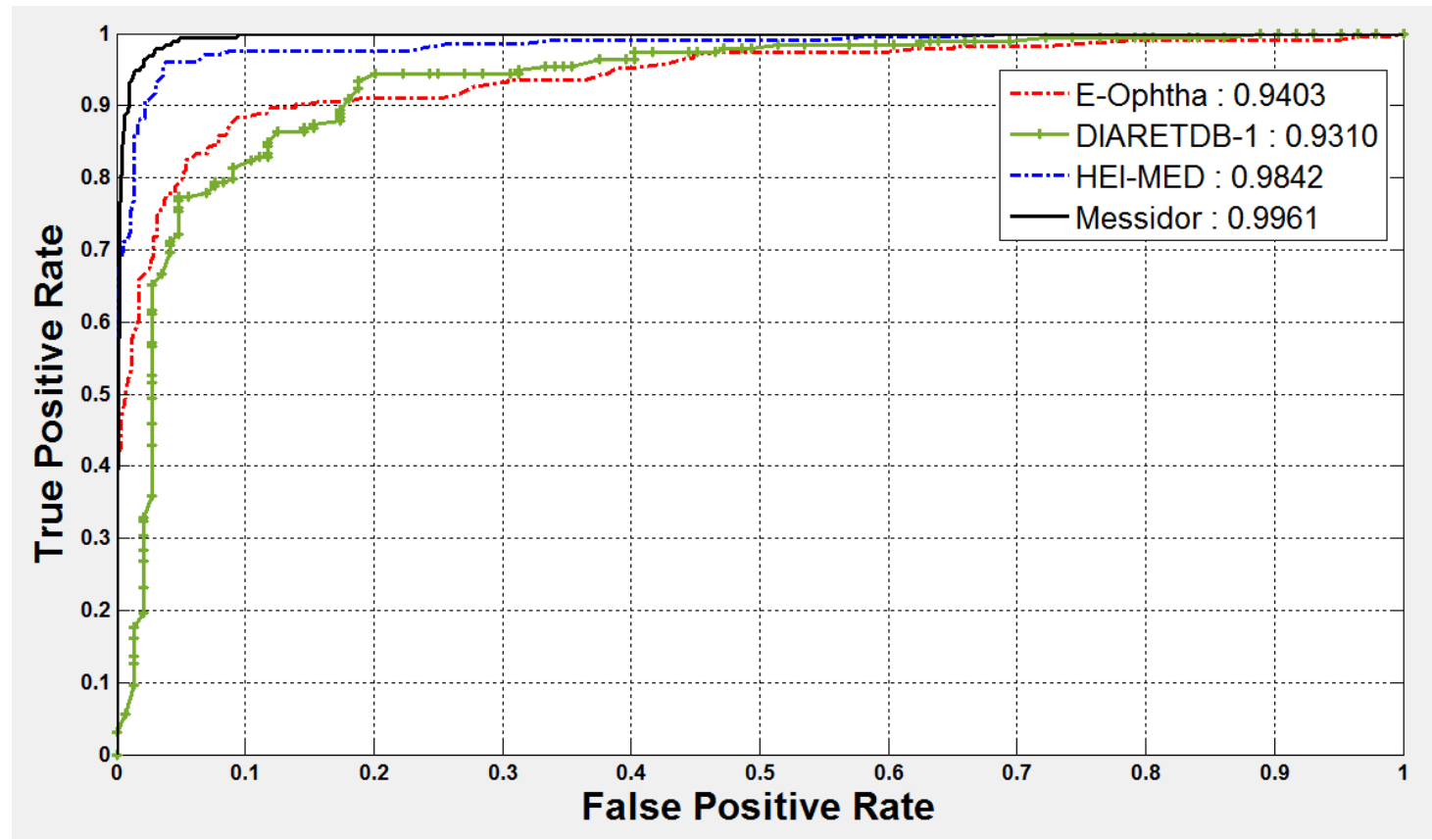

Fig. 11: ROC plots for different retinal image datasets

The methodology is implemented in Matlab R2015b, and is evaluated on Dell XPS 13 Corei7 with 8GB of RAM on Windows 8 Operating System. The performance metrics and processing time for each of the retinal image datasets are given in Table 5.

Table 5 : Exudates Classification performance measure on different retinal image datasets

\begin{tabular}{|l|c|c|c|c|c|c|c|}
\hline \multicolumn{1}{|c|}{ Database } & AUC & Acc & SN & SP & PPV & FDR & $\begin{array}{c}\text { Processing Time } \\
\text { (per image) }\end{array}$ \\
\hline DIARETDB1 & 0.9310 & 0.8772 & 0.9242 & 0.8125 & 0.8714 & 0.1286 & $72 \mathrm{sec}$ \\
\hline e-Ophtha EX & 0.9403 & 0.8925 & 0.8120 & 0.9460 & 0.9091 & 0.0909 & $96 \mathrm{sec}$ \\
\hline HEI-MED & 0.9842 & 0.9577 & 0.9463 & 0.9641 & 0.9372 & 0.0628 & $87 \mathrm{sec}$ \\
\hline Messidor & 0.9961 & 0.9836 & 0.9231 & 0.9903 & 0.9137 & 0.0863 & $77 \mathrm{sec}$ \\
\hline
\end{tabular}

\begin{tabular}{|l|c|c|}
\hline Database & Original Image & $\begin{array}{c}\text { Exudates Superimposed on Original } \\
\text { Image }\end{array}$ \\
\hline
\end{tabular}




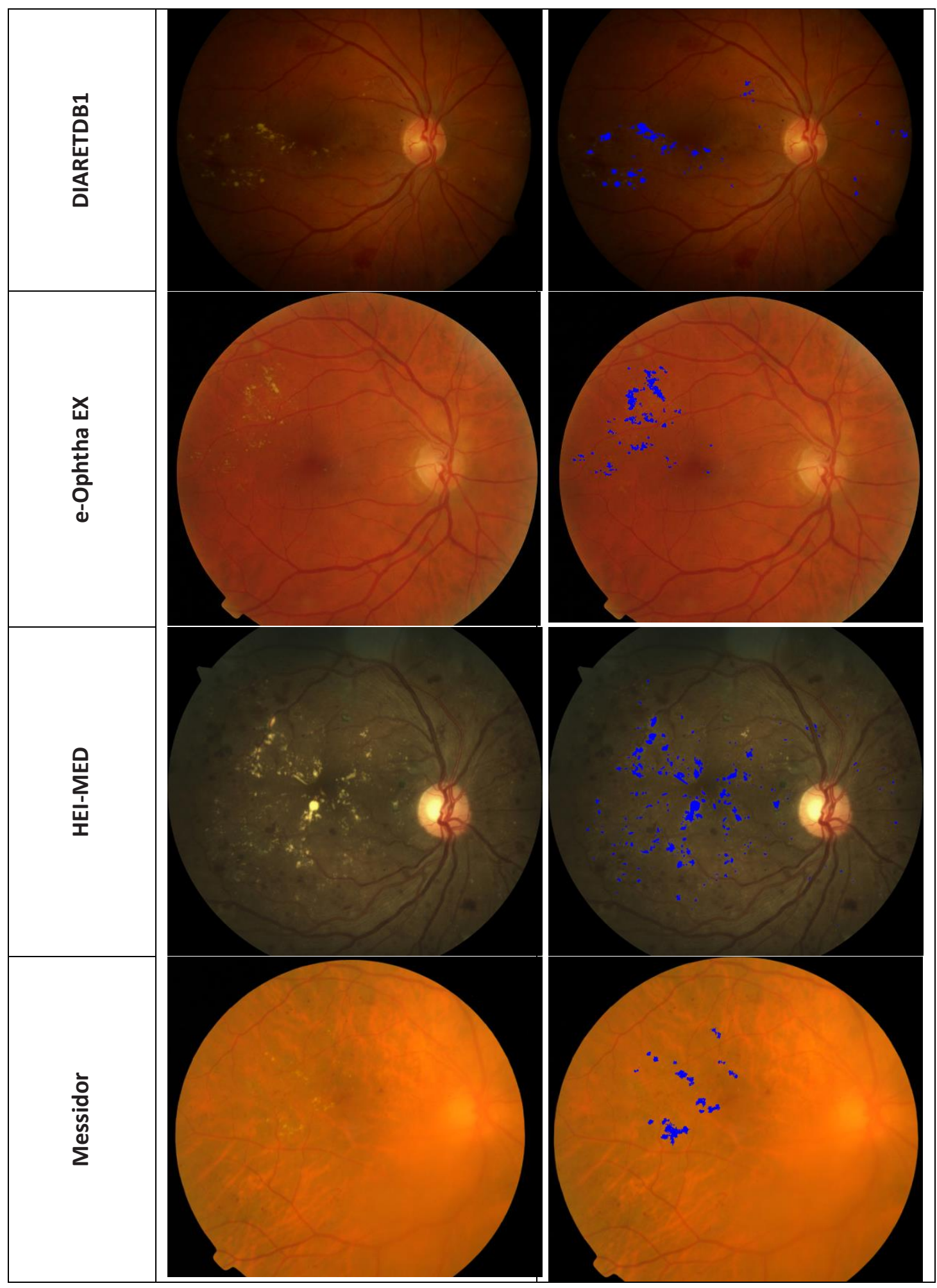

Fig. 12 : Sample exudate classification results on different retinal images

\subsection{Comparison with other Methods}

The comparison of presented method with previously reported methods in literature is difficult, due to the unavailability of a common retinal image-set and the performance metrics. The performance of 
proposed methodology has been evaluated at pixel level, by counting the number of correctly identified pixels as exudates and non-exudates in comparison with manually segmented exudates identified by the clinical experts. To the limit of our knowledge, this is one of the few exudate segmentation methods [16], where the pixel based evaluation has been reported. The performance measures of proposed methodology and existing techniques are compared in Table 6. The details about the number of images used for test and training for each retinal image dataset is illustrated in section 4.1. It has been observed that Jaafar et al [7] got an impressive average accuracy of $99 \%$. However, they have used 47 out of 89 images in their experimentation. We are unable to replicate their performance measure because the implementation of their methodology and the ground truths they have used are not available online. As mentioned earlier, most of the algorithms described in literature have not been evaluated on these databases, neither do these methodologies report all of the performance metrics reported by our algorithm.

Table 6: Comparison with other methods

\begin{tabular}{|l|l|l|l|l|l|l|}
\hline Database & Methodology & AUC & Acc & SN & SP & PPV \\
\hline \multirow{5}{*}{ DIARETDB1 } & Walter [5] & - & - & 0.76 & - & 0.59 \\
\cline { 2 - 7 } & Jaafar et al [7] & - & 0.99 & 0.89 & 0.993 & - \\
\cline { 2 - 7 } & Welfer [8] & - & - & 0.704 & 0.988 & 0.92 \\
\cline { 2 - 7 } & Harangi [10] & - & 0.82 & 0.86 & - & 0.84 \\
\cline { 2 - 7 } & Harangi [40] & - & - & 0.73 & - & 0.69 \\
\cline { 2 - 7 } & Proposed Method & $\mathbf{0 . 9 3 1 0}$ & $\mathbf{0 . 8 7 7 2}$ & $\mathbf{0 . 9 2 4 2}$ & $\mathbf{0 . 8 1 2 5}$ & $\mathbf{0 . 8 7 1 4}$ \\
\hline \multirow{5}{*}{ HEI-MED } & Ali et al. [9] & 0.83 & 0.82 & - & - & - \\
\cline { 2 - 7 } & Harangi et.al [10] & - & 0.86 & 0.87 & 0.86 & - \\
\cline { 2 - 7 } & Zhang et al. [16] & 0.94 & - & - & - & - \\
\cline { 2 - 7 } & Proposed Method & $\mathbf{0 . 9 8 4 2}$ & $\mathbf{0 . 9 5 7 7}$ & $\mathbf{0 . 9 4 6 3}$ & $\mathbf{0 . 9 6 4 1}$ & $\mathbf{0 . 9 3 7 2}$ \\
\hline \multirow{3}{*}{ e-Ophtha Ex } & Zhang et al. [16] & 0.95 & - & 0.74 & - & 0.79 \\
\cline { 2 - 7 } & Proposed Method & $\mathbf{0 . 9 4 0 3}$ & $\mathbf{0 . 8 9 2 5}$ & $\mathbf{0 . 8 1 2 0}$ & $\mathbf{0 . 9 4 6 0}$ & $\mathbf{0 . 9 0 9 1}$ \\
\hline \multirow{5}{*}{ Messidor } & Zhang et al.[16] & 0.93 & - & - & - & - \\
\cline { 2 - 7 } & Agurto [41] & 0.96 & 0.93 & 0.79 & 0.92 & - \\
\cline { 2 - 7 } & Proposed Method & $\mathbf{0 . 9 9 6 1}$ & $\mathbf{0 . 9 8 3 6}$ & $\mathbf{0 . 9 2 3 1}$ & $\mathbf{0 . 9 9 0 3}$ & $\mathbf{0 . 9 1 3 7}$ \\
\hline
\end{tabular}

\section{Conclusion}

We have presented and extensively evaluated an algorithm for localization and segmentation of exudates in retinal fundus images, based on an ensemble classifier of bagged decision trees. The methodology can successfully deal with images with uneven background illumination, variability in contrast during image acquisition, different background pigmentation and presence of artefacts. The proposed methodology performs exudate detection and candidate exudate region identification at fine grain and coarse levels to deal with the variability in size of exudate regions. Contextual information about exudates is used to refine the process of candidate exudate delineation, which has greatly reduced the false positives in segmented exudates. The exudates do not appear randomly in whole of the retinal. Generally they are not present in the region of main vascular arch. This contextual information is used to refine the candidate exudate pixels in such a way that the exudate candidates in the vascular arch region are filtered out. The feature vector is computed from the candidate exudate regions. The feature vector encodes information which can successfully quantify the variable sized 
exudates in the retinal images with multiple artefacts. An ensemble classifier of bootstrapped decision trees has been used to classify the candidate regions as exudates and non-exudates. To the best of our knowledge, this classic ensemble classifier has been utilized for the first time for pixel-level segmentation and classification of exudates in retinal images.

A significant feature of ensemble classifier of bagged decision trees is that the approximation of classifier's predictive power and the importance of a particular feature in classification can be computed during the classifier training without using the test data. Moreover, the optimal number of decision trees used to construct the ensemble classifier as well as the number of training sample can be reliably estimated during the classifier training, thus reducing the computational load with increased efficiency, as elaborated in section 3.5.2.

The performance of proposed methodology has been evaluated on four publically available databases with an array of performance metrics. Extensive quantitative evaluation of exudate segmentation is performed the obtained performance measures, robustness and accuracy are comparable with the state-of-the-art methodologies available in the literature.

The performance of the proposed exudate detection algorithm can be evaluated at two different levels. This assessment can be performed at the distinct exudate level when an accurate pixel level segmentation of exudates performed by clinical experts is available. This pixel level evaluation method is particularly interesting, when we are comparing different exudate detection algorithms. We have developed a software tool for clinical experts, which enables them to precisely create ground truths for exudates at pixel level interactively, as explained in section 3.6.

The second level of assessment is the evaluation at image level for the presence or absence of exudates. This image level evaluation is of importance from clinical aspect, for executing screening programs for early detection of DR in large population based studies. To the best of our knowledge, the proposed method is the first one that has been evaluated on four publicly available datasets simultaneously, with a number of performance metrics. Our contribution could be considered as a first step towards a common framework for the evaluation of different exudate segmentation algorithms and could be integrated into an image level evaluation framework.

Lastly, the demonstrated simplicity and computational speed in training/classification have made this ensemble-based supervised exudate segmentation method to be an appropriate tool for incorporation into an automated system for timely discovery of DR. We have already developed a fully automated software system named QUARTZ [32], which can extract a number of quantifiable measures from retinal vessel morphology. These measures are analyzed/studied by the epidemiologists and other medical/statistical experts in order to evaluate the association of retinal vessel abnormalities with other systemic diseases. In future, we aim to enhance the aforementioned software system and extend its functionality by incorporating a module for early DR detection in large population based studies. The presented method for reliable exudate localization and segmentation can be seen as a first step towards the development of a diabetic retinopathy detection module.

\section{Conflict of interest statement}


Authors disclose that they do not have any known conflict of interest.

\section{Acknowledgements}

The Authors are thankful to the teams of DIARETDB1, e-Optha Ex, HEI-MED and Messidor for maintaining and keeping these databases alive and making it easily accessible for public to carry on research.

\section{References}

[1] Jack J. Kanski, Brad Bowling, Clinical Ophthalmology: A Systematic Approach, 8th ed., Elsevier Health Sciences (UK), London, 2015.

[2] I.D. Federation, International Diabetic Federation Atlas; Chapter 3 Regional overviews, in, International Diabetic Federation, 2013, pp. 51-68.

[3] S. Philip, A.D. Fleming, K.A. Goatman, S. Fonseca, P. Mcnamee, G.S. Scotland, G.J. Prescott, P.F. Sharp, J.A. Olson, The efficacy of automated "disease/no disease" grading for diabetic retinopathy in a systematic screening programme, British Journal of Ophthalmology, 91 (2007) 1512-1517.

[4] W.M.D.W. Zaki, M.A. Zulkifley, A. Hussain, W.H.W.A. Halim, N.B.A. Mustafa, L.S. Ting, Diabetic retinopathy assessment: Towards an automated system, Biomedical Signal Processing and Control, 24 (2016) 72-82.

[5] T. Walter, J.C. Klein, P. Massin, A. Erginay, A contribution of image processing to the diagnosis of diabetic retinopathy-detection of exudates in color fundus images of the human retina, Medical Imaging, IEEE Transactions on, 21 (2002) 1236-1243.

[6] A. Sopharak, B. Uyyanonvara, S. Barman, T.H. Williamson, Automatic detection of diabetic retinopathy exudates from non-dilated retinal images using mathematical morphology methods, Computerized Medical Imaging and Graphics, 32 (2008) 720-727.

[7] H.F. Jaafar, A.K. Nandi, W. Al-Nuaimy, Detection of exudates in retinal images using a pure splitting technique, in: Engineering in Medicine and Biology Society (EMBC), 2010 Annual International Conference of the IEEE, IEEE, 2010, pp. 6745-6748.

[8] D. Welfer, J. Scharcanski, D.R. Marinho, A coarse-to-fine strategy for automatically detecting exudates in color eye fundus images, Computerized Medical Imaging and Graphics, 34 (2010) 228-235.

[9] S. Ali, D. Sidibé, K.M. Adal, L. Giancardo, E. Chaum, T.P. Karnowski, F. Mériaudeau, Statistical atlas based exudate segmentation, Computerized Medical Imaging and Graphics, 37 (2013) 358-368.

[10] B. Harangi, A. Hajdu, Automatic exudate detection by fusing multiple active contours and regionwise classification, Computers in biology and medicine, 54 (2014) 156-171. 
[11] M. Niemeijer, B. van Ginneken, S.R. Russell, M.S. Suttorp-Schulten, M.D. Abramoff, Automated Detection and Differentiation of Drusen, Exudates, and Cotton-wool Spots in Digital Color Fundus Photographs for Early Diagnosis of Diabetic Retinopathy IOVS-06-0996 accepted version, Investigative ophthalmology \& visual science, 48 (2007) 2260.

[12] D.F. Alan, P. Sam, A.G. Keith, J.W. Graeme, A.O. John, F.S. Peter, Automated detection of exudates for diabetic retinopathy screening, Physics in Medicine and Biology, 52 (2007) 7385.

[13] R. Acharya U, C. Chua, E.Y.K. Ng, W. Yu, C. Chee, Application of Higher Order Spectra for the Identification of Diabetes Retinopathy Stages, Journal of medical systems, 32 (2008) 481-488.

[14] M. García, C.I. Sánchez, M.I. López, D. Abásolo, R. Hornero, Neural network based detection of hard exudates in retinal images, Computer methods and programs in biomedicine, 93 (2009) 9-19.

[15] M.U. Akram, A. Tariq, S.A. Khan, M.Y. Javed, Automated detection of exudates and macula for grading of diabetic macular edema, Computer methods and programs in biomedicine, 114 (2014) 141152.

[16] X. Zhang, G. Thibault, E. Decencière, B. Marcotegui, B. Laÿ, R. Danno, G. Cazuguel, G. Quellec, M. Lamard, P. Massin, A. Chabouis, Z. Victor, A. Erginay, Exudate detection in color retinal images for mass screening of diabetic retinopathy, Medical Image Analysis, 18 (2014) 1026-1043.

[17] C. Pereira, L. Gonçalves, M. Ferreira, Exudate segmentation in fundus images using an ant colony optimization approach, Information Sciences, 296 (2015) 14-24.

[18] L. Giancardo, F. Meriaudeau, T.P. Karnowski, Y. Li, S. Garg, K.W. Tobin Jr, E. Chaum, Exudate-based diabetic macular edema detection in fundus images using publicly available datasets, Medical image analysis, 16 (2012) 216-226.

[19] C.I. Sánchez, M. Niemeijer, I. Išgum, A. Dumitrescu, M.S.A. Suttorp-Schulten, M.D. Abràmoff, B. van Ginneken, Contextual computer-aided detection: Improving bright lesion detection in retinal images and coronary calcification identification in CT scans, Medical image analysis, 16 (2012) 50-62.

[20] M.M. Fraz, P. Remagnino, A. Hoppe, B. Uyyanonvara, A.R. Rudnicka, C.G. Owen, S.A. Barman, Blood vessel segmentation methodologies in retinal images - A survey, Computer methods and programs in biomedicine, 108 (2012) 407-433.

[21] M.M. Fraz, A. Rudnicka, C. Owen, S. Barman, Delineation of blood vessels in pediatric retinal images using decision trees-based ensemble classification, International Journal of Computer Assisted Radiology and Surgery, 9 (2014) 795-811.

[22] M. Foracchia, E. Grisan, A. Ruggeri, Luminosity and contrast normalization in retinal images, Medical image analysis, 9 (2005) 179-190. 
[23] S.G. Vázquez, B. Cancela, N. Barreira, M.G. Penedo, M. Rodríguez-Blanco, M. Pena Seijo, G.C. Tuero, M.A. Barceló, M. Saez, Improving retinal artery and vein classification by means of a minimal path approach, Machine Vision and Applications, 24 (2013) 919-930.

[24] M.M. Fraz, A. Basit, S.A. Barman, Application of Morphological Bit Planes in Retinal Blood Vessel Extraction, Journal of Digital Imaging, (2012) 1-13.

[25] A. Basit, M.M. Fraz, Optic disc detection and boundary extraction in retinal images, Applied optics, 54 (2015) 3440-3447.

[26] K.W. Tobin, E. Chaum, V.P. Govindasamy, T.P. Karnowski, Detection of anatomic structures in human retinal imagery, Medical Imaging, IEEE Transactions on, 26 (2007) 1729-1739.

[27] J. Serra, Image Analysis and Mathematical Morphology, Academic Press, Inc, Orlando, FL, USA, 1983.

[28] M.M. Fraz, S.A. Barman, P. Remagnino, A. Hoppe, A. Basit, B. Uyyanonvara, A.R. Rudnicka, C.G. Owen, An approach to localize the retinal blood vessels using bit planes and centerline detection, Computer methods and programs in biomedicine, 108 (2012) 600-616.

[29] J. Sung, S.-Y. Bang, S. Choi, A Bayesian network classifier and hierarchical Gabor features for handwritten numeral recognition, Pattern Recognition Letters, 27 (2006) 66-75.

[30] R.C. Gonzalez, R.E. Woods, S.L. Eddins, Digital Image processing using MATLAB, 2nd edition ed., Gatesmark Publishing, 2009.

[31] M.M. Fraz, A.R. Rudnicka, C.G. Owen, D.P. Strachan, S.A. Barman, Automated Arteriole and Venule Recognition in Retinal Images using Ensemble Classification, in: 9th International Conference on Computer Vision Theory and Applications (VISAAP), Lisbon, Portugal, 2014.

[32] M.M. Fraz, R.A. Welikala, A.R. Rudnicka, C.G. Owen, D.P. Strachan, S.A. Barman, QUARTZ: Quantitative Analysis of Retinal Vessel Topology and size - An automated system for quantification of retinal vessels morphology, Expert Systems with Applications, 42 (2015) 7221-7234.

[33] R. Polikar, Ensemble based systems in decision making, Circuits and Systems Magazine, IEEE, 6 (2006) 21-45.

[34] L. Breiman, Bagging Predictors, Machine Learning, 24 (1996) 123-140.

[35] R. Polikar, Bootstrap - Inspired Techniques in Computation Intelligence, Signal Processing Magazine, IEEE, 24 (2007) 59-72. 
[36] T. Kauppi, V. Kalesnykiene, J.-K. Kamarainen, L. Lensu, I. Sorri, A. Raninen, R. Voutilainen, H. Uusitalo, H. Kälviäinen, J. Pietilä, The DIARETDB1 Diabetic Retinopathy Database and Evaluation Protocol, in: BMVC, 2007, pp. 1-10.

[37] E. Decencière, G. Cazuguel, X. Zhang, G. Thibault, J.-C. Klein, F. Meyer, B. Marcotegui, G. Quellec, M. Lamard, R. Danno, TeleOphta: Machine learning and image processing methods for teleophthalmology, IRBM, 34 (2013) 196-203.

[38] L. Giancardo, F. Meriaudeau, T.P. Karnowski, Y. Li, S. Garg, K.W. Tobin, E. Chaum, Exudate-based diabetic macular edema detection in fundus images using publicly available datasets, Medical image analysis, 16 (2012) 216-226.

[39] E. Decenciere, X. Zhang, G. Cazuguel, B. Laÿ, B. Cochener, C. Trone, P. Gain, J.-R. Ordónez-Varela, P. Massin, A. Erginay, Feedback on a publicly distributed image database: the MESSIDOR database, Image Analysis and Stereology, (2014) 231-234.

[40] B. Harangi, A. Hajdu, Detection of exudates in fundus images using a Markovian segmentation model, in: Engineering in Medicine and Biology Society (EMBC), 2014 36th Annual International Conference of the IEEE, 2014, pp. 130-133.

[41] C. Agurto, V. Murray, H. Yu, J. Wigdahl, M. Pattichis, S. Nemeth, E.S. Barriga, P. Soliz, A Multiscale Optimization Approach to Detect Exudates in the Macula, Biomedical and Health Informatics, IEEE Journal of, 18 (2014) 1328-1336. 